\title{
The diversity of RNAi and its applications
}

\author{
BioTechniques 40:S4-S5 (April 2006)
}

doi 10.2144/000112168

\begin{abstract}
Inhibiting the expression of genetic information has long been the domain of classical genetics. In recent years, some useful nucleic acid-based tools have been developed that allow studies of gene function in organisms that are not amenable to rapid genetic analyses. These tools include antisense oligomers, ribozymes, DNAzymes, and aptamers. Many useful analyses have been conducted with these reagents, and in some instances the reagents have been developed into therapeutics. Most recently, the discovery of an endogenous mechanism for regulation of gene expression, RNA interference (RNAi), has created a small revolution in functional genomics, which is rapidly spreading into therapeutics. RNAi is a highly conserved phenomenon in eukaryotes, apparently evolved as a defense mechanism against viral infections.
\end{abstract} Although RNAi was observed in plants more than a decade ago, it is triggered by short $(<25$ nucleotides) double-stranded duplexes processed from longer RNA duplexes. There are two major classes of these small RNAs, the microRNAs (miRNAs) and small interfering RNAs (siRNAs). miRNAs most often derive from longer precursor hairpin transcripts whose stems form imperfect Watson-Crick helices. The siRNAs are derived from double-stranded RNA (dsRNA) precursors with complete Watson-Crick base pairing, most often viral in origin. Both of these RNAs assemble with a core of proteins called the RNA-induced silencing complex, or RISC. The most prominent members of RISC are a family of proteins called Argonaute. One member of this family, Argonaute 2, has a catalytic domain that functions to cleave one strand of a duplex RNA, resulting in functional destruction of the cleaved strand. Other members of this family lack the catalytic domain and thus function by recruiting proteins that inhibit translation, possibly at the step of initiation. Functionally, miRNAs and siRNAs inhibit gene expression by different mechanisms. miRNAs most often bind to the $3^{\prime}$ untranslated region of target transcripts, forming incomplete Watson-Crick base pairs and blocking translation. siRNAs form perfect or near perfect Watson-Crick helices anywhere along a target mRNA and direct sequence-specific cleavage of the target. It is important to note that miRNAs and siRNAs are interchangeable, depending upon the extent of base pairing with the target mRNA. A third function of small RNAs is the silencing of gene expression at the level of transcription by signaling the formation of heterochromatin, termed transcriptional gene silencing (TGS). This mechanism is prominent in fission yeast, where it is involved in maintaining a heterochromatic state in centromeric regions, and in plants, in which TGS plays a prominent role as an anti-viral defense mechanism. Recent evidence has shown that TGS can be induced in mammalian cells, although its role in mammalian gene regulation is presently unknown.

This special supplement covers a wide variety of topics ranging from therapeutic potential of RNAi in combating human diseases to the role of RNAi in TGS and in suppressing diseaselinked messenger RNA (mRNA) isoforms. In the following section, we provide a brief introduction to each of the reviews.

This supplement starts with a review by Kevin Morris that describes advances in our understanding of RNAi-mediated TGS. TGS was first observed in tobacco plants, and subsequent studies showed that dsRNAs direct methylation of histones, resulting in a series of chromatin structural alterations, which ultimately lead to chromatin silencing. Recent studies confirmed that dsRNA-mediated TGS is present in Arabidopsis, Schizosaccharomyces pombe, and mammalian cells. The fact that siRNA can mediate gene silencing at the level of transcription has opened up new avenues in controlling gene expression by directing epigenetic changes in local chromatin structure. 
Transcripts produced by RNA polymerase II undergo processing before they serve as templates for protein synthesis. One of the most important steps in mRNA processing is the removal of the noncoding (intron) sequences from the precursor mRNA via pre-mRNA splicing. A highly sophisticated ribonucleoprotein assembly, known as the spliceosome, catalyzes intron removal and exon ligation and with the help of other regulatory proteins orchestrates alternative splicing that allows the production of different mRNA isoforms. An estimated 35\%-70\% of human genes generate transcripts that are alternatively spliced, and defects in this process are linked to numerous human diseases. The review by Rajesh Gaur overviews the fundamental process of RNA splicing, and discusses how alternatively spliced mRNA isoform(s) that are linked to human diseases can be the targets of RNAi-mediated knockdown.

One of the first proposed applications of RNAi was its use as an antiviral agent. Cells can be programmed with siRNAs that direct cleavage of viral transcripts, and in some cases cellular transcripts, thereby blocking viral gene expression and replication. John Rossi overviews antiHIV applications using RNAi and discusses therapeutic strategies and potential pitfalls for doing this.

Insulin resistance is characterized by a reduced ability of insulin to regulate glucose homeostasis in target tissues and can lead to many diseases ranging from cardiomyopathy to type II diabetes. Although the molecular basis of insulin resistance is poorly defined, altered expression of genes that mediate insulin signaling play a central role in insulin resistance. The main focus of the review by Cristina Rondinone is an evaluation of the therapeutic potential of RNAi in targeting genes that are linked to metabolic diseases, including the genes involved in insulin action in different tissues.

The emergence of resistance to newer antimalarial agents has posed a serious problem in the therapy of malaria caused by Plasmodium falciparum. According to World Health Organization estimates, approximately a half billion clinical cases of malaria are reported each year, leading to over one million deaths. Brown and Catteruccia have touched upon this devastating disease by first providing a detailed understanding of the life cycle of the malaria parasite, followed by a discussion of how RNAi can be applied to the identification of factors within the mosquito vector that support parasite development and maintenance. They also explore the potential for RNAi-mediated gene knockdown in P. falciparum for the identification of new drugs.

We hope that these reviews will not only capture the attention of researchers working in the specialized field(s), but will also be of broad general interest to those who wish to develop a basic understanding of RNAi. Finally, we would like to thank the authors for their contributions and the reviewers for their insightful recommendations.

—Rajesh K. Gaur and John J. Rossi, Guest Editors Beckman Research Institute of the City of Hope,

Duarte, $C A$ 